\title{
A pandemia de Covid-19 e seus impactos nos pacientes com Diabetes Mellitus
}

\author{
The Covid-19 pandemic and its impacts on patients with Diabetes Mellitus \\ La pandemia de Covid-19 y sus impactos en pacientes con Diabetes Mellitus
}

Recebido: 03/02/2022 | Revisado: 07/02/2022 | Aceito: 17/02/2022 | Publicado: 25/02/2022

\author{
Marcos Rogerio Silva Rosa \\ ORCID: https://orcid.org/0000-0003-3794-5529 \\ Universidade Federal de São João del Rei, Brasil \\ rogerinhomarcos02@gmail.com \\ Ana Carolina Oliveira Gonçalves \\ ORCID: https://orcid.org/0000-0001-9000-4491 \\ Universidade Federal de São João del Rei, Brasil \\ anacarolina@ufsj.edu.br
}

\begin{abstract}
Resumo
Introdução: Após a detecção do SARS-CoV-2 na cidade de Wuhan, na China, a Organização Mundial da Saúde (OMS) reconheceu a infecção pelo novo Coronavírus como um grave problema de saúde pública e a caracterizou como uma pandemia. Objetivo: Este estudo objetiva analisar como a pandemia do novo Coronavírus afetou pacientes adultos e idosos com diabetes mellitus e se a literatura é capaz de observar a diferença de desfechos de acordo com as medidas de controle adotadas em cada país. Método: Foi realizada uma revisão de literatura a partir das bases de dados eletrônicas PubMed, Biblioteca Virtual de Saúde (BVS) e Google Scholar utilizando-se as palavras chaves "diabetes mellitus", "Covid-19", "Sars Cov 2", "Coronavírus". Resultados: Analisando os 19 estudos incluídos nesta revisão, observa-se que o diabetes é um importante fator de risco para Covid-19, considerando que as evidências sugerem maior risco de quadros graves da doença com maiores chances de necessidade de ventilação mecânica invasiva e mortalidade, além de observar que países que adotaram confinamento rigoroso tiveram pouco impacto no controle glicêmico, ao passo que os países que não adotaram deixaram seus pacientes mais expostos a piora do controle glicêmico. Conclusão: Diante do exposto, pode-se afirmar que pacientes com diabetes estão mais expostos às complicações mais graves como internação na UTI, ventilação mecânica invasiva o que implica no aumento dos índices de morbidade e mortalidade dos mesmos. Além disso, observa-se que um pobre controle glicêmico estava associado a quadros mais graves, bem como a capacidade de autogestão dos pacientes com relação à doença.
\end{abstract}

Palavras-chave: Diabetes mellitus; Covid-19; Coronavírus; SARS-CoV-2.

\begin{abstract}
Introduction: After the detection of SARS-CoV-2 in the city of Wuhan, China, the World Health Organization (WHO) recognized the new Coronavirus infection as a serious public health problem and in March 2020 characterized it as a pandemic. Given this situation, some population groups were considered at high risk for severe cases of Covid-19, including patients with Diabetes mellitus, a clinical condition of high prevalence in the world population. Objective: This study analyzed how the new Coronavirus pandemic affected adult and elderly patients with diabetes mellitus and whether the literature was able to observe the difference in outcomes according to the control measures adopted in each country. Method: A literature review was carried out from the electronic databases PubMed, Virtual Health Library (VHL) and Google Scholar using the keywords "Diabetes mellitus", "Covid-19", "Sars Cov 2", "Coronavirus", "Adults". Results: There were 19 studies included in this review. It was observed that diabetes is an important risk factor for Covid-19, with a higher probability of severe disease with the need for invasive mechanical ventilation and higher mortality. The strict and structured confinement had less impact on glycemic control, whereas in countries that did not adopt it, it was possible to observe a deterioration in the glycemic control of patients. Conclusion: Patients with diabetes, especially those with poor glycemic control, are more exposed to the most serious complications of Covid-19. Adequate compliance with social isolation measures was a protective factor for good glycemic control.
\end{abstract}

Keywords: Diabetes mellitus; Covid-19; Coronavirus; SARS-CoV-2.

\section{Resumen}

Introducción: Tras la detección del SARS-CoV-2 en la ciudad de Wuhan, China, la Organización Mundial de la Salud (OMS) reconoció la nueva infección por Coronavirus como un grave problema de salud pública y en marzo de 2020 la caracterizó como una pandemia. Ante esta situación, algunos grupos poblacionales fueron considerados de alto riesgo para casos severos de Covid-19, incluidos los pacientes con Diabetes mellitus, una condición clínica de alta prevalencia en la población mundial. Objetivo: Este estudio analizó cómo la nueva pandemia de coronavirus afectó a pacientes adultos y ancianos con diabetes mellitus y si la literatura pudo observar la diferencia en los resultados según las medidas de control adoptadas en cada país. Método: Se realizó una revisión de la literatura de las bases de datos electrónicas 
PubMed, Virtual Health Library (BVS) y Google Scholar utilizando las palabras clave "Diabetes mellitus", "Covid19", "Sars Cov 2", "Coronavirus", “Adultos". . Resultados: se incluyeron 19 estudios en esta revisión. Se observó que la diabetes es un factor de riesgo importante para Covid-19, con mayor probabilidad de enfermedad grave con necesidad de ventilación mecánica invasiva y mayor mortalidad. El confinamiento estricto y estructurado tuvo menor impacto en el control glucémico, mientras que en los países que no lo adoptaron se pudo observar un deterioro en el control glucémico de los pacientes. Conclusión: Los pacientes con diabetes, especialmente aquellos con mal control glucémico, están más expuestos a las complicaciones más graves del Covid-19. El adecuado cumplimiento de las medidas de aislamiento social fue un factor protector para un buen control glucémico.

Palabras clave: Diabetes mellitus; Covid-19; Coronavirus; SARS-CoV-2.

\section{Introdução}

Em dezembro de 2019, foi detectado um novo vírus, o SARS-CoV-2 na cidade de Wuhan, localizada na área central da China (Mohamadian et al., 2021). Em março de 2020 a Organização Mundial da Saúde (OMS) reconheceu a infecção pelo novo Coronavírus como um grave problema de saúde pública, caracterizando-se como uma pandemia. Segundo o boletim epidemiológico da OMS de 14 de outubro de 2021, são mais de 239 milhões de casos confirmados e 4.871 .841 mortes em todo o mundo (OMS,2021).

Uma vez infectado, o indivíduo com Covid-19 apresenta um quadro de Síndrome Respiratória Aguda, podendo, em alguns casos, resultar em importante comprometimento pulmonar e até mesmo óbito. O SARS-CoV-2 é transmitido de forma direta, por contato ou transmissão aérea através do contato com aerossóis, inalação de gotículas, tosse e espirro, sendo que as membranas oculares e nasais estão entre os modos mais comuns de disseminação (Umakanthan et al., 2020). O mecanismo de infecção do SARS-CoV-2 acontece pela penetração do vírus na célula através do receptor da enzima conversora de angiotensina 2 ECA2). Ele infecta predominantemente as vias aéreas inferiores e se liga à ECA2 nas células epiteliais alveolares, replica-se rapidamente e desencadeia uma forte resposta imunológica, resultando em uma descarga de citocinas e danos ao tecido pulmonar (Channappanavar \& Perlman, 2017). Sem tratamento farmacológico efetivo, as estratégias de contenção da disseminação do vírus envolvem hábitos como o uso de máscaras, higienização das mãos e a manutenção do distanciamento social. Em função da proporção de casos e da alta transmissibilidade do vírus, medidas de isolamento social também foram adotadas como forma de conter o avanço da pandemia. Estas medidas envolviam restrição de circulação, fechamento de atividades comerciais e industriais consideradas como não essenciais, entre outras (Jayaweera et al., 2020). Um esforço mundial foi despendido para o desenvolvimento de vacinas capazes de combater a doença. Segundo a OMS, até o dia 14 de outubro de 2021, mais de 6 bilhões de doses de vacina já foram administradas (OMS,2021).

Durante o curso da pandemia de Covid-19, alguns fatores de risco para desenvolvimento de quadros graves da doença foram identificados como, por exemplo, a presença de condições clínicas como diabetes mellitus (DM), hipertensão arterial sistêmica (HAS) ou doenças cardíacas, além de outros fatores como idade avançada e baixa função imunológica, asma e Doença Pulmonar Obstrutiva Crônica (DPOC) (Gao et al., 2021). O DM é uma doença de alta prevalência na população mundial (Gao et al., 2021). O curso natural da Covid-19 tende a ser favorável para a maioria dos pacientes, porém pacientes com doença crônica, como diabetes, tendem a apresentar piores desfechos em consequência da expressão de ECA2, o receptor de entrada do SARS-CoV-2, que está aumentada nesses pacientes nos pulmões e em outros tecidos. Tendo em vista que a regulação positiva está associada à ativação das células endoteliais, inflamação crônica e resistência à insulina, agrava-se a resposta inflamatória e promove à disfunção da barreira alveolar-capilar (Gao et al., 2021). Por isso destaca-se a importância de priorizar esses pacientes na educação e empoderamento sobre as estratégias de prevenção e também como prioridade de vacinação (Landstra \& de Koning, 2021). Diante do exposto, o presente estudo analisou como a pandemia do novo Coronavírus afetou pacientes adultos e idosos com diabetes mellitus tipo 1 e tipo 2 e se a literatura foi capaz de observar a diferença de desfechos de acordo com o rigor das medidas de controle da pandemia adotadas em cada país. 


\section{Metodologia}

Trata-se de uma revisão bibliográfica narrativa, exploratória e descritiva. A seleção dos estudos foi realizada a partir das bases de dados eletrônicas: U.S. National Library of Medicine (PubMed) e Biblioteca Virtual em Saúde (BVS). Os descritores “diabetes mellitus", "Covid-19", "Sars Cov 2", "Coronavírus" foram definidos a partir de Descritores em Ciências da Saúde (DECS): Diabetes Mellitus, Covid 19, Coronavírus, Sars Cov 2; e combinados com o operador booleano “AND”. Foram incluídos estudos publicados entre dezembro de 2019 a junho de 2021 nacionais ou internacionais, com texto completo disponível, publicados em português, inglês ou espanhol, com qualquer desenho de estudo descrito no Quadro 1. A remoção de duplicatas foi feita através da checagem do título e dos autores e a triagem dos estudos foi feita através da leitura dos resumos. Estudos que não contemplavam o assunto foram excluídos.

Quadro 1: Definição dos níveis de evidência dos estudos de acordo com "The Oxford 2011 Levels of Evidence"

\section{Nivel de evidência}

nível 1 - evidências resultantes da rev isão sistemática de ensaios clínicos randomizados

nivel 2 - ev idências resultantes de ensaio randomizado ou estudo observacional com efeito dramático

nível 3 - evidências resultantes de estudo de coorte / acompanhamento controlado não randomizado; nível 4 - evidências resultantes de estudos de série de casos, caso-controle ou historicamente controlados

nivel 5 - evidências resultantes de raciocinio baseado em mecanismo

Fonte: Adaptado de Oxford Centre for Evidence-Based Medicine 2011 Levels of Evidence (elaborado pelo autor).

\section{Resultados}

Ao final da busca nas bases de dados foram recuperados aproximadamente 2307 registros. Após a remoção de duplicatas, exclusão de artigos não disponíveis na íntegra e não relacionados ao tema foram incluídos 19 estudos nesta revisão, com predomínio do idioma inglês de estudos descritivos/qualitativos conforme apresentado na figura 1. Foi realizada leitura na íntegra de todos os artigos que atenderam aos critérios de inclusão descritos e, durante essa fase, variáveis Autores, Ano, País, Objetivo, tipo de estudo, principais resultados foram coletados e descritos no Quadro 2. 
Figura 1: Fluxograma das fases utilizadas para a seleção dos artigos.

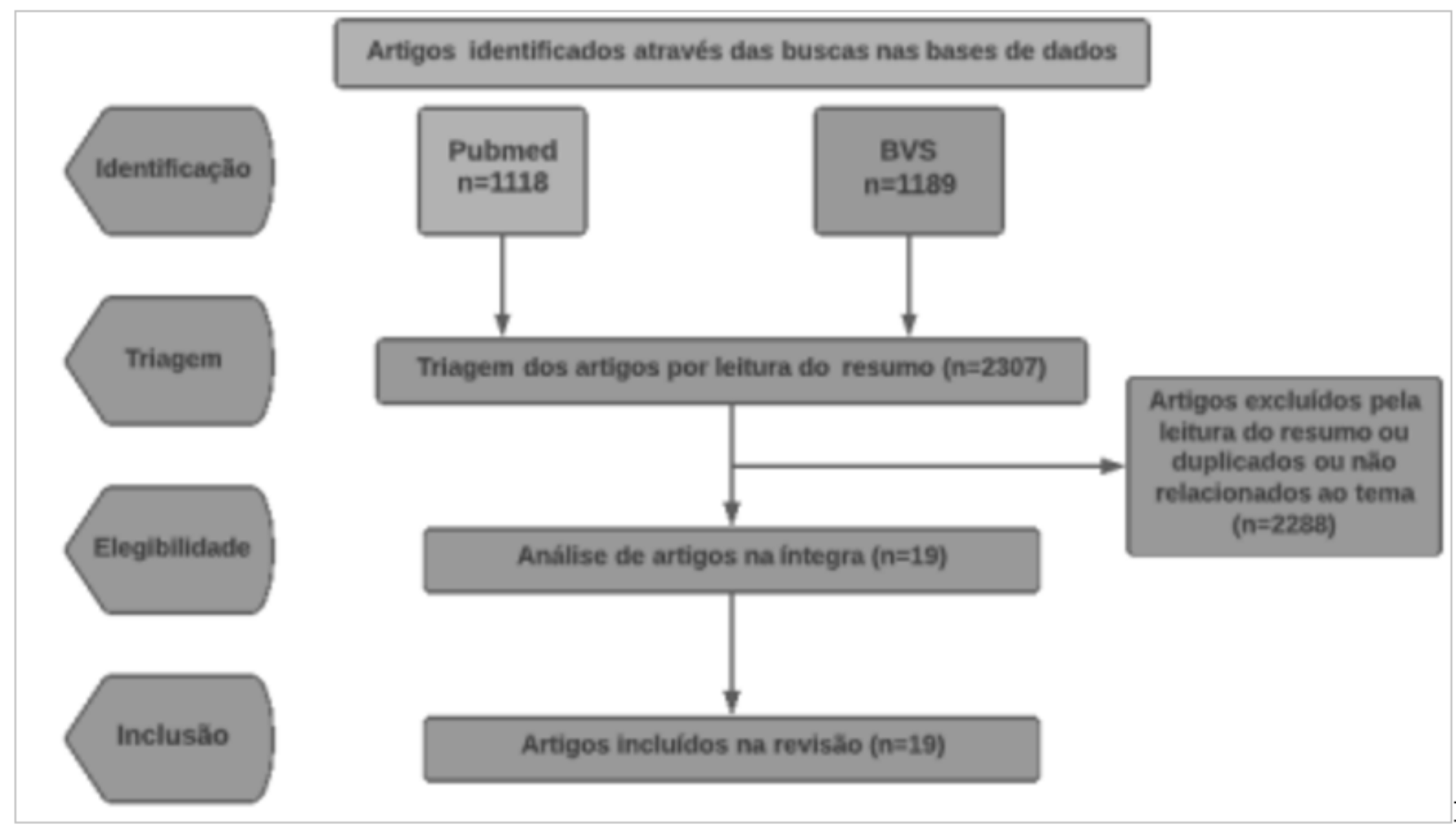

Fonte: Elaborado pelos autores.

Quadro 2: Síntese dos estudos que abordam diabetes mellitus e Covid-19.

\begin{tabular}{|c|c|c|c|}
\hline $\begin{array}{l}\text { Autor Principal } \\
\text { AnoPaís }\end{array}$ & Objetivo & $\begin{array}{l}\text { Tipo de estudo } \\
\text { Nível de } \\
\text { evidência }\end{array}$ & Resultado \\
\hline $\begin{array}{c}\text { Halla Kaminska } 2021 \\
\text { Polônia }\end{array}$ & $\begin{array}{l}\text { Investigar o diabetes mellitus (DM) } \\
\text { sobre a mortalidade hospitalar em } \\
\text { pacientes com Covid-19, através de } \\
\text { uma revisão sistemática e meta } \\
\text { análise. }\end{array}$ & $\begin{array}{l}\text { Revisão sistemática e } \\
\text { Meta-análise } \\
\text { Nível } 1\end{array}$ & $\begin{array}{l}\text { Quadro grave de Covid-19 em paciente com } \\
34,8 \% \text { contra } 22,8 \% \text { no grupo não-DM. Os } \\
\text { pacientes com DM apresentaram } 21,3 \% \text { de } \\
\text { taxa de mortalidade com maior frequência de } \\
\text { complicações. Os não-DM apresentaram } 6,1 \% \\
\text { de taxa de mortalidade. }\end{array}$ \\
\hline $\begin{array}{l}\text { Jing Wu } \\
2020 \\
\text { China }\end{array}$ & $\begin{array}{l}\text { Avaliar a influência do diabetes na } \\
\text { gravidade e letalidade da infecção por } \\
\text { SARS-COV-2. }\end{array}$ & $\begin{array}{l}\text { Meta-análise mista } \\
\text { Nível } 2\end{array}$ & $\begin{array}{l}\text { Do total de pacientes analisados ( } 66 \text { - } 22 \text { com } \\
\text { DM e } 44 \text { sem DM), } 31,82 \% \text { dos pacientes com } \\
\text { diabetes foram diagnosticados como casos } \\
\text { graves de Covid- } 19 \text {, conta } 9,09 \% \text { no grupo } \\
\text { sem diabetes, com um risco } 2,95 \text { vezes maior } \\
\text { de fatalidade em comparação com aqueles } \\
\text { pacientes sem diabetes. }\end{array}$ \\
\hline $\begin{array}{l}\text { Zeng-Hong } \\
2020 \\
\text { China }\end{array}$ & $\begin{array}{l}\text { Explorar a mortalidade entre diabetes } \\
\text { e Covid-19, analisando e avaliando a } \\
\text { confiabilidade das evidências. }\end{array}$ & $\begin{array}{l}\text { Meta-análise mista } \\
\text { Nível } 2\end{array}$ & $\begin{array}{l}\text { Com um Odds Ratio agrupado de } 1,75 \text { (IC95\% } \\
1,31-2,36 ; \mathrm{P}=0,0002 \text { ), os resultados exibem } \\
\text { uma estreita relação entre diabetes e } \\
\text { mortalidade de Covid-19. Confiabilidade dos } \\
\text { dados (FEN): nenhuma heterogeneidade } \\
\text { apareceu nos estudos. Após análises de } \\
\text { sensibilidade mantiveram-se os principais } \\
\text { resultados. }\end{array}$ \\
\hline
\end{tabular}




\begin{tabular}{|c|c|c|c|}
\hline $\begin{array}{c}\text { Alamin Alkundi, Ibrahim } \\
\text { Mahmoud } \\
2020 \\
\text { Reino Unido }\end{array}$ & $\begin{array}{l}\text { Descrever os desfechos e as } \\
\text { características clínicas dos pacientes } \\
\text { Covid-19 com diabetes e sem } \\
\text { diabetes internados em um } \\
\text { hospital da Inglaterra de março a } \\
\text { maio de 2020. }\end{array}$ & $\begin{array}{l}\text { Estudo } \\
\text { transversal } \\
\text { retrospectivo } \\
\text { Nível } 2\end{array}$ & $\begin{array}{l}\text { Pacientes internados com COVID } \\
\text { apresentaram maior tempo de permanência na } \\
\text { internação, quando comparados àqueles sem } \\
\text { DM. Pacientes com quadros de cetoacidose } \\
\text { diabética tiveram maior probabilidade de } \\
\text { sobreviver. }\end{array}$ \\
\hline $\begin{array}{l}\text { Anjali Verma, Rajesh } \\
\text { Rajput } \\
2020 \\
\text { Índia }\end{array}$ & $\begin{array}{l}\text { Avaliar o impacto do isolamento no } \\
\text { controle glicêmico empacientes com } \\
\text { Diabetes Mellitus tipo } 1 \text { (DM1) }\end{array}$ & $\begin{array}{l}\text { Estudo } \\
\text { Observacional } \\
\text { Nível } 2\end{array}$ & $\begin{array}{l}\text { Dentre os } 52 \text { pacientes, } 15,3 \% \text { apresentavam } \\
\text { episódios hipoglicêmicos e } 36,5 \% \\
\text { apresentavam estado hiperglicêmico. } 26,9 \% \\
\text { esqueceram alguma dose de inslina e } 36,5 \% \\
\text { relataram que o monitoramento da glicose não } \\
\text { foi feito rotineiramente. Já } 17,4 \% \text { não estavam } \\
\text { em conformidade com a dieta durante o } \\
\text { "lockdown". O valor médio da HbA1c durante } \\
\text { o isolamento foi de } 10( \pm 1,5 \%) \text { contra } 8,8( \pm \\
1,3 \%) \text { na fase pré-isolamento }(\mathrm{p}<0,05) \text {. }\end{array}$ \\
\hline $\begin{array}{l}\text { You-Ping Deng } \\
2021 \\
\text { China }\end{array}$ & $\begin{array}{l}\text { Analisar a associação do diabetes } \\
\text { com mortalidade e gravidade entre } \\
\text { pacientes hospitalizados com Covid- } \\
19 \text { em Wuhan, uma área central da } \\
\text { China. }\end{array}$ & $\begin{array}{l}\text { Estudo } \\
\text { retrospectivo } \\
\text { Nível } 2\end{array}$ & $\begin{array}{l}\text { Os pacientes com diabetes apresentaram } \\
\text { proporções significativamente maiores de } \\
\text { casos críticos e mortalidade hospitalar. Pela } \\
\text { curva de Kaplan-Meier, observa-se uma } \\
\text { tendência para menor sobrevida em pacientes } \\
\text { com DM comparados com pacientes sem DM. }\end{array}$ \\
\hline $\begin{array}{l}\text { Ortega } \\
2021 \\
\text { Espanha }\end{array}$ & $\begin{array}{l}\text { Analisar o risco de complicações } \\
\text { hospitalares graves em pacientes } \\
\text { internados por Covid-19 e diabetes } \\
\text { mellitus (DM), }\end{array}$ & $\begin{array}{l}\text { Estudo } \\
\text { transversal } \\
\text { Nível } 2\end{array}$ & $\begin{array}{l}\text { A taxa de mortalidade hospitalar geral foi de } \\
18,6 \%(\mathrm{~N}=301) \text {, sendo } 26,3 \% \text { entre os } \\
\text { pacientes com } \mathrm{DM} \text { e } 11,3 \% \text { entre aqueles sem } \\
\text { DM. O DM foi associado de forma } \\
\text { independente a óbito ou ventilação mecânica } \\
\text { invasiva. }\end{array}$ \\
\hline $\begin{array}{c}\text { Barrett } \\
2021 \\
\text { Estados Unidos }\end{array}$ & $\begin{array}{l}\text { Analisar se pacientes sem diabetes } \\
\text { possuem risco de desfechos graves } \\
\text { quando comparado a pacientes com } \\
\text { diabetes mellitus tipo } 1 \text { (DM1) ou } \\
\text { com diabetes mellitus tipo } 2 \text { (DM2). }\end{array}$ & $\begin{array}{l}\text { Estudo } \\
\text { Retrospectivo } \\
\text { Nível } 2\end{array}$ & $\begin{array}{l}\text { Em comparação com pacientes sem diabetes, } \\
\text { um risco absoluto } 21 \% \text { maior de internação } \\
\text { em unidades de terapia intensiva ou ventilação } \\
\text { mecânica invasiva (UTI/MV) foi associado } \\
\text { aos pacientes DM1. O DM1 quando } \\
\text { comparado com o DM2, foi associado a um } \\
\text { risco absoluto } 9 \% \text { maior de UTI/MV, mas sem } \\
\text { notar diferença na mortalidade. }\end{array}$ \\
\hline $\begin{array}{c}\text { Sharif } \\
2021 \\
\text { Bangladesh }\end{array}$ & $\begin{array}{l}\text { Apurar se o prognóstico e desfechos } \\
\text { graves de saúde entre pacientes com } \\
\text { Covid-19 sofre impacto do diabetes e } \\
\text { das doenças cardiovasculares (DCV). }\end{array}$ & $\begin{array}{l}\text { Estudo } \\
\text { retrospectivo } \\
\text { Nível } 2\end{array}$ & $\begin{array}{l}\text { Foram detectados } 40 \% \text { ( } 318 \text { de } 799 \text { ) de taxa de } \\
\text { letalidade em pacientes com Covid- } 19 \text {. Das } \\
318 \text { mortes, } 74,5 \% \text { foram detectadas em } \\
\text { pacientes com diabetes e } 90,6 \% \text { em pacientes } \\
\text { com DCV. Vale ressaltar que a taxa de } \\
\text { letalidade foi mais alta em pacientes com } \\
\text { diabetes (195), seguido por DCV(184), e por } \\
\text { Covid-19(94); e paralelamente as maiores } \\
\text { chances de risco de letalidade foram } \\
\text { detectadas em pacientes com Covid-19, DCV } \\
\text { e diabetes. }\end{array}$ \\
\hline $\begin{array}{l}\text { Ugliara Barone } \\
2020 \\
\text { Brasil }\end{array}$ & $\begin{array}{l}\text { Avaliar se os pacientes com diabetes } \\
\text { conseguiram manter o controle } \\
\text { glicêmico durante as medidas de } \\
\text { isolamento social durante a pandemia } \\
\text { de Covid-19. }\end{array}$ & $\begin{array}{l}\text { Estudo } \\
\text { observacional } \\
\text { Nível } 2\end{array}$ & $\begin{array}{l}\text { Durante a pandemia, nos estados de Bahia, } \\
\text { Goiás e Pernambuco menos de } 50 \% \text { dos } \\
\text { entrevistados apresentaram maior } \\
\text { variabilidade ou maior glicemia. O estado de } \\
\text { Goiás possui o maior percentual }(35,48 \%) \text { dos } \\
\text { indivíduos que receberam medicamentos por } \\
\text { três meses, obtendo um dos menores } \\
\text { percentuais de deterioração da glicemia com } \\
47,17 \% \text {. Vale ressaltar que houve adiamento } \\
\text { das consultas e/ou exames laboratoriais nos } \\
\text { estados de Minas Gerais, Rio de Janeiro e São } \\
\text { Paulo. }\end{array}$ \\
\hline
\end{tabular}




\begin{tabular}{|c|c|c|c|}
\hline $\begin{array}{l}\text { Khare } \\
2020 \\
\text { Índia }\end{array}$ & $\begin{array}{l}\text { Avaliar se confinamento domiciliar é } \\
\text { um fator que interfere no controle } \\
\text { glicêmico em pacientes com diabetes. }\end{array}$ & $\begin{array}{l}\text { Estudo } \\
\text { observacional } \\
\text { Nível } 2\end{array}$ & $\begin{array}{l}\text { Considerando os } 143 \text { pacientes com diabetes } \\
\text { que participaram do estudo, } 39,16 \% \text { dos } \\
\text { pacientes relataram piora da hiperglicemia e } \\
\text { necessitando de adição de medicamentos para } \\
\text { controle da glicemia e } 2,09 \% \text { pacientes } \\
\text { relataram eventos hipoglicêmicos e } \\
\text { medicamentos foram retirados. }\end{array}$ \\
\hline $\begin{array}{l}\text { Huiqing } \\
2020 \\
\text { China }\end{array}$ & $\begin{array}{l}\text { Analisar a associação entre diferentes } \\
\text { graus de hiperglicemia e o risco de } \\
\text { mortalidade por todas as causas entre } \\
\text { pacientes com Covid-19 } \\
\text { hospitalizados no Hospital Union em } \\
\text { Wuhan, China. }\end{array}$ & $\begin{array}{l}\text { Estudo } \\
\text { retrospectivo } \\
\text { Nível } 2\end{array}$ & $\begin{array}{l}\text { Pacientes com diabetes recém-diagnosticado } \\
\text { obtiveram o maior percentual de admissão na } \\
\text { unidade de terapia intensiva (UTI) com } \\
11,7 \% \text {; o mesmo valor foi obtido para } \\
\text { admissão com ventilação mecânica invasiva } \\
\text { (VMI), acompanhado por pacientes com } \\
\text { diabetes conhecido }(4,1 \% ; 9,2 \%) \text { e pacientes } \\
\text { com hiperglicemia }(6,2 \% ; 4,7 \%) \text {, quando } \\
\text { comparados com pacientes com glicemia } \\
\text { normal }(1,5 \% ; 2,3 \%) \text {. }\end{array}$ \\
\hline $\begin{array}{c}\text { Benedetta Maria } 2020 \\
\text { Itália }\end{array}$ & $\begin{array}{l}\text { Relatar o impacto do estado de } \\
\text { confinamento quase completo em } \\
\text { vista da pandemia do Covid-19 no } \\
\text { controle da glicose entre pessoas com } \\
\text { diabetes tipo 1 (DM1). }\end{array}$ & $\begin{array}{l}\text { Estudo } \\
\text { observacional } \\
\text { multicêntrico } \\
\text { Nível } 2\end{array}$ & $\begin{array}{l}\text { Os } 33 \text { pacientes com DM1 monitoravam seus } \\
\text { níveis de glicemia capilar. O controle } \\
\text { glicêmico global foi melhor durante os } \\
\text { primeiros } 7 \text { dias do confinamento, comparado } \\
\text { com as semanas anteriores, para } 20 \text { pacientes } \\
\text { que estavam em casa. Já dos } 13 \text { pacientes que } \\
\text { continuaram trabalhando, não foi observado } \\
\text { nenhuma alteração das medidas de controle } \\
\text { glicêmico. }\end{array}$ \\
\hline $\begin{array}{c}\text { Alharthi, Sahar K } 2021 \\
\text { Arábia Saudita }\end{array}$ & $\begin{array}{l}\text { Examinar o impacto do confinamento } \\
\text { no controle glicêmico em indivíduos } \\
\text { com DM1 utilizando monitoramento } \\
\text { contínuo de glicose (CGM), e } \\
\text { também analisar se consultas de } \\
\text { telemedicina influenciaram neste } \\
\text { controle glicêmico. }\end{array}$ & $\begin{array}{l}\text { Estudo } \\
\text { retrospectivo } \\
\text { Nível } 2\end{array}$ & $\begin{array}{l}\text { Não observou-se alterações significativas nas } \\
\text { métricas do CGM durante o confinamento nos } \\
\text { pacientes que não participaram de } \\
\text { atendimentos telemedicina, porém os que } \\
\text { participaram das consultas melhoraram as } \\
\text { métricas como a glicose média de } 180 \text { a } 159 \\
\text { mg/dl, e a HbA1c } 7,7 \% \text { para } 7,2 \% \text {. }\end{array}$ \\
\hline $\begin{array}{c}\text { Mesa } \\
2020 \\
\text { Espanha }\end{array}$ & $\begin{array}{l}\text { Examinar através do monitoramento } \\
\text { autônomo e contínuo de glicose } \\
\text { (CGM), o impacto do confinamento } \\
\text { no perfil glicêmico de pacientes com } \\
\text { DM1 propensos à hipoglicemia. }\end{array}$ & $\begin{array}{l}\text { Estudo } \\
\text { observacional } \\
\text { Nível } 2\end{array}$ & $\begin{array}{l}\text { A faixa de glicemia foi } 70-180 \mathrm{mg} / \mathrm{dL} \text {, o } \\
\text { parâmetro HbA1c melhorou de forma } \\
\text { significativa. Vale ressaltar que o tempo de } \\
\text { hipoglicemia permaneceu inalterado. }\end{array}$ \\
\hline $\begin{array}{l}\text { Eugene Merzon } \\
2020 \\
\text { Israel }\end{array}$ & $\begin{array}{l}\text { Descrever a associação entre os } \\
\text { níveis de hemoglobina glicada } \\
\text { (HbA1c) pré-infecção e a } \\
\text { hospitalização por Covid-19. }\end{array}$ & $\begin{array}{l}\text { Estudo de coorte } \\
\text { observacional } \\
\quad \text { Nível } 3\end{array}$ & $\begin{array}{l}\text { Controlando várias condições clínicas } \\
\text { anteriores, a análise multivariada demonstra } \\
\text { que o único parâmetro associado a um risco } \\
\text { significativamente aumentado de } \\
\text { hospitalização foi } \mathrm{HbAlc} \geq 9 \% \text {. }\end{array}$ \\
\hline $\begin{array}{l}\text { Holman } \\
2020 \\
\text { Reino Unido }\end{array}$ & $\begin{array}{l}\text { Analisar a relação entre fatores de } \\
\text { risco e morte relacionada a Covid- } 19 \\
\text { em uma coorte viva entre o período } \\
\text { de janeiro a maio de } 2020 \text {. }\end{array}$ & $\begin{array}{l}\text { Estudo de coorte } \\
\text { observacional } \\
\quad \text { Nível } 3\end{array}$ & $\begin{array}{l}\text { As mortes semanais mais do que dobraram nos } \\
\text { grupos DM1 e DM2, ultrapassando a variação } \\
\text { esperada. Das } 265.090 \text { pessoas com DM } 1 \text { e } \\
2.889 .210 \text { pessoas com DM2, transcorreram } \\
\text { respectivamente } 418 \text { e } 9.377 \text { mortes } \\
\text { relacionadas ao Covid-19. }\end{array}$ \\
\hline $\begin{array}{l}\text { Borzouei } \\
2021 \\
\text { Irã }\end{array}$ & $\begin{array}{l}\text { Investigar os preditores de morte em } \\
\text { pacientes Covid- } 19 \text { sem diabetes em } \\
\text { comparação com pacientes Covid-19 } \\
\text { com diabetes. }\end{array}$ & $\begin{array}{c}\text { Estudo de Caso controle } \\
\text { Nível } 4\end{array}$ & $\begin{array}{l}\text { Pelo modelo de regressão logística ajustada, } \\
\text { demonstra-se que pacientes Covid- } 19 \text { com } \\
\text { diabetes, idade avançada, alto nível de ureia e } \\
\text { de fosfatase foram relacionados ao aumento da } \\
\text { chance de morte de forma significativa. }\end{array}$ \\
\hline $\begin{array}{l}\text { Kok Hoe Chan } \\
2020 \\
\text { Estados Unidos }\end{array}$ & $\begin{array}{l}\text { Explorar os desfechos da infecção } \\
\text { por Covid-19 em pacientes com } \\
\text { cetoacidose diabética (DKA) e estado } \\
\text { hiperglicêmico hiperosmolar (HHS). }\end{array}$ & $\begin{array}{l}\text { Série de Casos } \\
\text { Nível } 4\end{array}$ & $\begin{array}{l}\text { Notificam-se } 6 \text { pacientes que apresentaram } \\
\text { Covid-19 com DKA/HHS combinados, uma } \\
\text { característica marcante foi que a DKA/HHS } \\
\text { combinado foi a apresentação inicial do } \\
\text { Covid-19. }\end{array}$ \\
\hline
\end{tabular}

Fonte: Elaborado pelos autores. 


\section{Discussão}

Diante do exposto no Quadro 2, evidencia-se que o diabetes é um importante fator de risco para Covid-19, considerando que as evidências sugerem maior risco de quadros graves da doença com maiores chances de necessidade de ventilação mecânica invasiva e mortalidade (Borzouei et al., 2021; Ortega et al., 2021). Esse resultado corrobora com Merzon et al (2021) que evidenciou que valores maiores de HbA1c também podem estar relacionados a piores desfechos no quadro de Covid-19. Além disso idade avançada, sexo masculino, coexistência de comorbidades e função renal comprometida também estão relacionados a um maior risco de desfechos graves (Barrett et al., 2021; Deng et al., 2021; England et al., n.d.; Li et al., 2020; Sharif et al., 2021; J. Wu et al., 2020; Z. hong Wu et al., 2021). Assim pode-se inferir que o controle glicêmico e os processos de educação para a autogestão do diabetes são parte importante para a redução dos riscos a que pacientes com diabetes estão expostos durante a pandemia de Covid-19.

Em relação ao impacto das medidas de isolamento social no controle glicêmico dos pacientes com diabetes, os achados expõem que entre os pacientes com DM1, as medidas de restrição de circulação tiveram um menor impacto no controle glicêmico, quando comparados aos pacientes com DM2, que segundo os achados tiveram uma piora dos parâmetros glicêmicos (Alharthi et al., 2021; Biamonte et al., 2021; Bonora et al., 2020; Mesa et al., 2020; Tanji et al., 2021). Segundo Bonora et al (2020); Lepelletier et al (2020) e Minuto et al (2021) a diferença encontrada entre os dois grupos pode ser compreendida quando analisamos a capacidade do autogerenciamento da doença. No DM1 não foi observado grande impacto no controle glicêmico. $\mathrm{O}$ que pode ser explicado pelo fato destes pacientes serem diagnosticados na infância ou na juventude e, portanto, possuírem suporte familiar no manejo da doença tanto em relação às aplicações de insulina como aos cuidados gerais com o diabetes como monitoramento rigoroso da glicemia e educação sobre a doença, reforçando a importância do sistema de apoio familiar para o bom o controle glicêmico. Assim, pode-se relacionar que nos países que adotaram um confinamento domiciliar rigoroso e bem planejado, o automonitoramento da glicemia capilar, a adaptação das atividades relacionadas ao trabalho para a forma remota, a implementação consultas por telemedicina e manutenção da atividade física, apresentaram efeitos benéficos no perfil glicêmico e na gestão da diabetes na população adulta (Alharthi et al., 2021; Bonora et al., 2020; Mesa et al., 2020). Em contrapartida, nos países que não adotaram lockdown rigoroso, o não planejamento da logística de saúde para os pacientes com comorbidades e a não adaptação do trabalho para as atividades remotas acarretaram uma deterioração do perfil glicêmico. Pacientes relataram que tiveram dificuldades para acessar seus suprimentos médicos mensalmente, resultando por vezes em estados hiperglicêmicos ou episódios de hipoglicemia. (Biamonte et al., 2021; Kaminska et al., 2021; Khare \& jindal, 2020; Tanji et al., 2021; Ugliara Barone et al., 2020). Além disso o estresse psicológico, aumento do consumo de alimentos, deterioração do humor, diminuição da atividade física, mudanças no estilo de vida, dificuldade em obter orientação médica também estão relacionados à um impacto negativo nos valores de glicemia e IMC de pacientes com diabetes (khare \& jindal, 2020; Tanji et al., 2021).

Neste sentido, destaca-se a importância do farmacêutico atuante na saúde pública (SUS) inserido na equipe multiprofissional como agente fundamental na promoção do controle glicêmico dos pacientes com diabetes através da adoção de estratégias como cartilhas educativas, empoderamento farmacoterapêutico para os pacientes, visando melhorar o estado de saúde dos mesmos e ajudando-os a ter maior domínio sobre sua condição (Aquino et al., 2019). Esse resultado corrobora com estudo de Biamonte et al (2021) e Liu et al (2020) que demonstra que o farmacêutico também pode atuar redigindo orientação de serviço profissional para farmácia e farmacêuticos, estabelecendo formulários de medicamentos de emergência com objetivo de monitorar e resolver a escassez de medicamentos, e também estabelecer serviço remoto visando educar a população sobre prevenção de infecções e gerenciamento de doenças, além de participar de ensaios clínicos e atuar na avaliação e gerenciamento de medicamentos. Assim, evidencia-se a importância de criar estratégias para manutenção do cuidado longitudinal a pacientes com diabetes no contexto do isolamento e após ele ser superado. Dentre as estratégias adotadas no âmbito do Sistema Único de Saúde(SUS), fomentar uso da telessaúde com intuito de facilitar a interação entre pacientes e a equipe multiprofissional, 
mostrando melhora a adesão ao tratamento para o paciente, além de reduzir riscos de infecção e economizar tempo e custos do tratamento; além disso, o incentivo a utilização de aplicativos relacionado à educação em diabetes associados a um treinador virtual seria importante, pois através dessa associação é possível enfrentar as barreiras comuns de tratamento e outras disparidades de saúde, além de fornecer suporte individualizado em tempo real em qualquer lugar e qualquer hora do dia (Ramchandani, 2019).

Esse trabalho demonstra que a maioria dos estudos incluídos na revisão possuem nível de evidência 2, o que pode ser explicado que pelo contexto da pandemia houve dificuldades para se conduzir estudos com metodologias mais robustas. Embora saiba-se destas limitações, as evidências apontadas devem ser consideradas para embasar tomada de decisão em saúde, considerando a alta prevalência do DM e sua relação com a gravidade dos quadros de Covid-19.

\section{Conclusão}

Com base nos achados, é possível afirmar que pacientes com diabetes estão mais expostos às complicações mais graves como internação na UTI, ventilação mecânica invasiva o que implica no aumento dos índices de morbidade e mortalidade dos mesmos. Notou-se também que países em que o isolamento social aconteceu sem planejamento, os pacientes com DM tiveram uma piora do controle glicêmico. Observou-se ainda que um mal controle glicêmico estava associado a quadros mais graves. Neste sentido, é notória a necessidade do desenvolvimento de práticas capazes de promover a educação em diabetes, perpassando por temas como adesão a farmacoterapia, automonitoramento da glicemia capilar, identificação e gestão de sintomas, entre outras práticas. Neste cenário, o farmacêutico se destaca como um profissional capacitado e que, de acordo com as evidências atuais, é capaz de promover este cuidado de forma efetiva.

\section{Referências}

Alharthi, S. K., Alyusuf, E. Y., Alguwaihes, A. M., Alfadda, A., \& Al-Sofiani, M. E. (2021). The impact of a prolonged lockdown and use of telemedicine on glycemic control in people with type 1 diabetes during the COVID-19 outbreak in Saudi Arabia. Diabetes Research and Clinical Practice, $173,108682$.

Alkundi, A., Mahmoud, I., Musa, A., \& Naveed, S. (2020). Clinical characteristics and outcomes of COVID-19 hospitalized patients with diabetes in the United Kingdom: A retrospective single centre study.

Aquino, J. A., Baldoni, A. O., Di Lorenzo Oliveira, C., Cardoso, C. S., de Figueiredo, R. C., \& Sanches, C. (2019). Pharmacotherapeutic empowerment and its effectiveness in glycemic control in patients with Diabetes Mellitus. Diabetes and Metabolic Syndrome: Clinical Research and Reviews, 13(1), $137-142$.

Barrett, C. E., Park, J., Kompaniyets, L., Baggs, J., Cheng, Y. J., Zhang, P., Imperatore, G., \& Pavkov, M. E. (2021). Intensive Care Unit Admission, Mechanical Ventilation, and Mortality Among Patients With Type 1 Diabetes Hospitalized for COVID-19 in the U.S. Diabetes Care, 44(8), 1788-1796.

Biamonte, E., Pegoraro, F., Carrone, F., Facchi, I., Favacchio, G., Lania, A. G., Mazziotti, G., \& Mirani, M. (2021). Weight change and glycemic control in type 2 diabetes patients during COVID-19 pandemic: the lockdown effect. Endocrine, 72(3), 604-610.

Bonora, B. M., Boscari, F., Avogaro, A., Bruttomesso, D., \& Fadini, G. P. (2020). Glycaemic Control Among People with Type 1 Diabetes During Lockdown for the SARS-CoV-2 Outbreak in Italy. Diabetes Therapy, 11(6), 1369-1379.

Borzouei, S., Mohammadian-khoshnoud, M., Omidi, T., Bashirian, S., Bahreini, F., Heidarimoghadam, R., \& Khazaei, S. (2021). Predictors of COVID-19 related death in diabetes patients: A case-control study in Iran. Diabetes and Metabolic Syndrome: Clinical Research and Reviews, 15(4).

Chan, K. H., Thimmareddygari, D., Ramahi, A., Atallah, L., Baranetsky, N. G., \& Slim, J. (2020). Clinical characteristics and outcome in patients with combined diabetic ketoacidosis and hyperosmolar hyperglycemic state associated with COVID-19: A retrospective, hospital-based observational case series. Diabetes Research and Clinical Practice, 166, 108279.

Channappanavar, R., \& Perlman, S. (2017). Pathogenic human coronavirus infections: causes and consequences of cytokine storm and immunopathology. Seminars in Immunopathology, 39(5), 529-539.

Deng, Y., Xie, W., Liu, T., Wang, S., Zan, Y., Wang, M., Meng, X., Zheng, J., Xiong, H., \& Fu, X. (2021). Association of diabetes with severity and mortality in hospitalized patients with COVID-19 in Wuhan, China: a single-centered, retrospective study. Archives of Endocrinology and Metabolism.

England, N. H. S., Holman, N., Keefe, J. O., \& Curley, M. (n.d.). Pr ep rin pe er r ev ed Pr ep rin t $n$ ot pe er ed.

Gao, Y. dong, Ding, M., Dong, X., Zhang, J. jin, Kursat Azkur, A., Azkur, D., Gan, H., Sun, Y. li, Fu, W., Li, W., Liang, H. ling, Cao, Y. yuan, Yan, Q., Cao, C., Gao, H. yu, Brüggen, M. C., van de Veen, W., Sokolowska, M., Akdis, M., \& Akdis, C. A. (2021). Risk factors for severe and critically ill COVID-19 patients: A review. In Allergy: European Journal of Allergy and Clinical Immunology (Vol. 76, Issue 2). 
Howick, J (2011). OCEBM Levels of Evidence Working Group. "The Oxford 2011 Levels of Evidence http://www.cebm.net/index.aspx?o=5653

Jayaweera, M., Perera, H., Gunawardana, B., \& Manatunge, J. (2020). Since January 2020 Elsevier has created a COVID-19 resource centre with free information in English and Mandarin on the novel coronavirus COVID- 19. The COVID-19 resource centre is hosted on Elsevier Connect, the company 's public news and information. Envronmental Research, 188(January), 1-18.

Kaminska, H., Szarpak, L., Kosior, D., Wieczorek, W., Szarpak, A., Al-Jeabory, M., Gawel, W., Gasecka, A., Jaguszewski, M. J., \& Jarosz-Chobot, P. (2021). Impact of diabetes mellitus on in-hospital mortality in adult patients with COVID-19: a systematic review and meta-analysis. Acta Diabetologica, 58(8), 11011110 .

khare, J., \& jindal, S. (2020). Observational study on Effect of Lock Down due to COVID 19 on glycemic control in patients with Diabetes: Experience from Central India. Diabetes and Metabolic Syndrome: Clinical Research and Reviews, 14(6), 1571-1574.

Landstra, C. P., \& de Koning, E. J. P. (2021). COVID-19 and Diabetes: Understanding the Interrelationship and Risks for a Severe Course. Frontiers in Endocrinology, 12(June), 1-18.

Lepelletier.D Michael J. Smart, Robert B. Noland, G. B. (2020). Repercusión del confinamiento por COVID-19 sobre el control glucémico en nĩ̃nos y adolescentes con diabetes mellitus tipo 1. Ann Oncol, January, 19-21.

Li, H., Tian, S., Chen, T., Cui, Z., Shi, N., Zhong, X., Qiu, K., Zhang, J., Zeng, T., Chen, L., \& Zheng, J. (2020). Newly diagnosed diabetes is associated with a higher risk of mortality than known diabetes in hospitalized patients with COVID-19. Diabetes, Obesity and Metabolism, 22(10), 1897-1906.

Liu, S., Luo, P., Tang, M., Hu, Q., Polidoro, J. P., Sun, S., \& Gong, Z. (2020). Providing pharmacy services during the coronavirus pandemic. International Journal of Clinical Pharmacy, 42(2), 299-304.

Merzon, E., Green, I., Shpigelman, M., Vinker, S., Raz, I., Golan-Cohen, A., \& Eldor, R. (2021). Haemoglobin A1c is a predictor of COVID-19 severity in patients with diabetes. Diabetes/Metabolism Research and Reviews, 37(5).

Mesa, A., Viñals, C., Pueyo, I. I., Roca, D., Vidal, M., Giménez, M., \& Conget, I. (2020). The impact of strict COVID-19 lockdown in Spain on glycemic profiles in patients with type 1 Diabetes prone to hypoglycemia using standalone continuous glucose monitoring. Diabetes Research and Clinical Practice, 167, $1-5$.

Minuto, N., Bassi, M., Montobbio, C., Vinci, F., Mercuri, C., Perri, F. N., Cabri, M., Calevo, M. G., d'Annunzio, G., \& Maghnie, M. (2021). The Effect of Lockdown and Physical Activity on Glycemic Control in Italian Children and Young Patients With Type 1 Diabetes. Frontiers in Endocrinology, 12(July), 19 .

Mohamadian, M., Chiti, H., Shoghli, A., Biglari, S., Parsamanesh, N., \& Esmaeilzadeh, A. (2021). COVID-19: Virology, biology and novel laboratory diagnosis. Journal of Gene Medicine, 23(2), 1-11.

Ortega, E., Corcoy, R., Gratacòs, M., Cos Claramunt, F. X., Mata-Cases, M., Puig-Treserra, R., Real, J., Vlacho, B., Castelblanco, E., Domingo, P., Khunti, K., Franch-Nadal, J., \& Mauricio, D. (2021). Risk factors for severe outcomes in people with diabetes hospitalised for COVID-19: A cross-sectional database study. BMJ Open, 11(7), 1-10.

Ramchandani, N. (2019). Virtual Coaching to Enhance Diabetes Care. Diabetes Technology and Therapeutics, 21(S2), S2-48-S2-51.

Sharif, N., Ahmed, S. N., Opu, R. R., Tani, M. R., Dewan, D., Daullah, M. U., Shanto, R. I., Parvez, A. K., Talukder, A. A., \& Dey, S. K. (2021). Prevalence and impact of diabetes and cardiovascular disease on clinical outcome among patients with COVID-19 in Bangladesh. Diabetes and Metabolic Syndrome: Clinical Research and Reviews, 15(3), 1009-1016.

Tanji, Y., Sawada, S., Watanabe, T., Mita, T., Kobayashi, Y., Murakami, T., Metoki, H., \& Akai, H. (2021). Impact of COVID-19 pandemic on glycemic control among outpatients with type 2 diabetes in Japan: A hospital-based survey from a country without lockdown. Diabetes Research and Clinical Practice, 176, 108840 .

Ugliara Barone, M. T., Harnik, S. B., Chaluppe, M., Vieira de Luca, P., Ngongo, B., Pedrosa, H. C., Pirolo, V., Franco, D. R., Malta, D. C., \& Giampaoli, V. (2020). Decentralized COVID-19 measures in Brazil were ineffective to protect people with diabetes. Diabetes and Metabolic Syndrome: Clinical Research and Reviews, 14(6), 1973-1978.

Umakanthan, S., Sahu, P., Ranade, A. V., Bukelo, M. M., Rao, J. S., Abrahao-Machado, L. F., Dahal, S., Kumar, H., \& Kv, D. (2020). Origin, transmission, diagnosis and management of coronavirus disease 2019 (COVID-19). Postgraduate Medical Journal, 96(1142), 753-758.

Verma, A., Rajput, R., Verma, S., Balania, V. K. B., \& Jangra, B. (2020). Impact of lockdown in COVID 19 on glycemic control in patients with type 1 Diabetes Mellitus. Diabetes and Metabolic Syndrome: Clinical Research and Reviews, 14(5), 1213-1216.

WHO Coronavírus (COVID-19) Dashboard, (2021). Referência dos dados epidemiológicos atualizados https://covid19.who.int/

Wu, J., Zhang, J., Sun, X., Wang, L., Xu, Y., Zhang, Y., Liu, X., \& Dong, C. (2020). Influence of diabetes mellitus on the severity and fatality of SARS-CoV2 (COVID-19) infection. Diabetes, Obesity and Metabolism, 22(10), 1907-1914.

Wu, Z. hong, Tang, Y., \& Cheng, Q. (2021). Diabetes increases the mortality of patients with COVID-19: a meta-analysis. Acta Diabetologica, 58(2), 139-144. 